\title{
Essais
}

ESSAIS

Revue interdisciplinaire d'Humanités

\section{Du rôle du contexte pour l'interprétation des lexèmes employés familièrement}

Jackie Schön

\section{(2) OpenEdition \\ 1 Journals}

\section{Édition électronique}

URL : http://journals.openedition.org/essais/2917

DOI : $10.4000 /$ essais. 2917

ISSN : 2276-0970

Éditeur

École doctorale Montaigne Humanités

\section{Édition imprimée}

Date de publication : 15 juin 2017

Pagination : 129-148

ISBN : 979-10-97024-02-4

ISSN : 2417-4211

\section{Référence électronique}

Jackie Schön, « Du rôle du contexte pour l'interprétation des lexèmes employés familièrement », Essais [En ligne], 12 | 2017, mis en ligne le 12 octobre 2020, consulté le 25 octobre 2020. URL : http:// journals.openedition.org/essais/2917 ; DOI : https://doi.org/10.4000/essais.2917 


\title{
Du rôle du contexte pour l'inter- prétation des lexèmes employés familièrement
}

\author{
Jackie Schön
}

"L'exemple n'est pas un autre moyen d'enseigner, c'est le seul.»

Albert Einstein

\section{Le cadrage}

À l'origine de la réflexion, un questionnement : comment un étranger apprenant notre langue comprendra-t-il un énoncé tel que "Qu'est-ce que tu fabriques avec ce chameau qui n'a jamais produit que des navets?" ? De quels repères et de quels outils dispose-t-il pour l'interpréter correctement ? N'aura$\mathrm{t}$-il d'autre recours que le dictionnaire ? Certes, le contexte, - tant l'environnement linguistique que l'énonciatif - lui fournira de précieuses indications pour accéder au sens adapté aux circonstances mais quels éléments linguistiques et quels éléments situationnels l'aideront-ils efficacement et selon quelles modalités ?

C'est à de telles interrogations que cette approche vise à proposer des réponses, aussi partielles ou provisoires fussent-elles. Jusqu'à quel point convient-il d'analyser les composantes d'un événement énonciatif ? Peut-on poser des limites généralisables à leur examen ; par ex. est-il, systématiquement, pertinent de connaître la température ou le degré d'hygrométrie de l'air environnant chaque énonciation pour leur juste interprétation?

\section{La démarche}

Il m'est impossible, dans le cadre d'une contribution, de citer la totalité des auteurs qui, au fil du temps, ont nourri ma recherche sur les emplois familiers des lexèmes. Ce disant, j'encours les reproches de la part de mes pairs, reproches que je ne conteste nullement, ne trouvant comme éventuelle - et bien faible - justification que l'entrée de nos universités dans l'ère du numérique laquelle ne manque pas de bousculer quelque peu nos traditions. 
Cependant, parmi les ouvrages travaillés, il est une étude, non encore caduque, qui aura joué comme un point de départ au présent exposé : tout en y traitant entièrement du thème défini pour le volume collectif, elle l'aborde selon une méthode totalement différente de la mienne. Il s'agit du livre «Le contexte en sémantique ${ }^{1}$ " du linguiste fonctionnaliste ${ }^{2}$ Mortéza Mahmoudian lequel cerne la notion de contexte à tous ses niveaux, la décompose en ses divers éléments et met en relief leurs mutuelles intrications.

L'auteur, procédant par enquêtes, fonde ses définitions sur leurs résultats et hiérarchise les sens des éléments linguistiques d'après l'ordre que lui fournissent ses informateurs dotés de leurs différents " savoirs ${ }^{3}$ cumulés. Ainsi, parmi les sens du mot chien, pris en exemple ${ }^{4}$, se rencontre celui de "personne méprisable " sans plus de conditions, sans que jamais n'intervienne le terme même d'acception 5 ce qui, concernant le complexe contextuel, me paraît curieux mais venons-en plutôt à la méthode employée ici.

À l'opposé de la démarche prudente et scientifiquement irréprochable de M. Mahmoudian, je ne m'appuie que sur mes compétences de locutrice native, compétences adossées, cependant, à des dictionnaires - de langue de préférence - parmi les plus courants et d'éditions pas trop anciennes. Je considère et utilise ce type de dictionnaires comme témoignages relativement neutres d'un certain état de la langue. Tout exemple retenu ici, ne le sera qu'après vérification du fait qu'il figure dans les dictionnaires accompagné d'un indice de restriction de ses emplois, soit fam. ou pop. ou autre (cf. infra, p. 126).

Mes exemples sont de ceux qui se profèrent et s'entendent communément, bribes de discours prédicatifs comparables à celles que présentent les dictionnaires. Ajoutons enfin que, dans l'optique adoptée, un seul contreexemple pèsera autant, sinon plus, qu'une longue liste d'exemples.

J'éviterai de suivre le parcours diachronique des lexèmes; la façon dont, peut-être, l'image du dépendeur d'andouilles a pu en arriver, après réduction de l'expression, à ce que le lexème andouille renvoie aujourd'hui à niais, imbécile ${ }^{6}$ sort du sujet. Une telle fantaisie semble régner dans de prétendues explications de l'émergence des sens familiers des mots qu'il vaut mieux, souvent, s'abstenir d'en privilégier aucune.

1 Mortéza Mahmoudian : Le contexte en sémantique, Peeters, Louvain-la neuve, 1997.

2 Courant dans lequel je me reconnais.

3 Je reprends, ici, le terme employé par l'auteur, terme dont il spécifiera les valeurs au long de son ouvrage selon leur niveau d'appartenance respective : savoir linguistique, savoir situationnel et savoir culturel.

4 Cf. p. 74-75 de l'ouvrage cité.

5 Étant entendu que qui dit acception dit sens en emploi.

6 Le Petit Robert, éd. 2000 : Sens 2 : FAM. Niais, imbécile. Quelle andouille, ce type! Faire l'andouille : faire l'imbécile ou simuler la naïveté. 
J'exclus également de mon objet les locutions ainsi que tout syntagme figé - totalement ou partiellement - ; ils présentent des caractéristiques contextuelles si spécifiques qu'ils justifient des approches ciblées ${ }^{7}$.

Enfin, la langue, en tant que système normé, est exposée à toutes sortes de distorsions et transgressions pour se plier aux multiples exigences - besoins, désirs ou fantasmes... - de ses utilisateurs. De fait, elle porte en elle ses pouvoirs d'ornementation, de figuration (i.e. de faire image), de symbolisation et aussi de manipulation ou de classification sociale de ses usagers. Les procédés utilisés pour obtenir des effets déterminés ont été identifiés et dûment répertoriés depuis l'antiquité ${ }^{8}$. Dans les cas qui nous occupent, nous avons aussi affaire à des figures de rhétorique, des substitutions de sens des mots mais, la différence capitale entre les tropes classiques et les emplois familiers des lexèmes réside en ce que, si les premiers sont volontaires, les seconds reposent sur des principes qui échappent largement à la conscience.

La mise en lumière de ces principes sous-jacents au processus de familiarisation des lexèmes constitue l'essentiel des pages qui suivent. Dans la mesure où l'objectif est de dévoiler ce qui se passe inconsciemment chez le locuteur lorsqu'il emploie une tournure familière, la question de son intentionnalité ne trouve pas sa place à cet endroit?

En revanche, les substitutions sémantiques, tant les rhétoriques que les familières, ressortissent à des écarts, des transgressions par rapport à la Norme mais de quelle norme s'agit-il ? De celle qui concerne le système de la langue (donnant, alors, lieu à des transgressions internes) ou de celle qu' instaurent les conventions sociales, les convenances? C'est ce qu'il nous reste à développer et je prendrai l'échange comme cadre opératoire de ma réflexion.

\section{De l'usage des dictionnaires}

Un parler devient familier soit par l'emploi de lexèmes qualifiés de familiers dès leur entrée dans les dictionnaires tout-venant ${ }^{10}$, soit par l'emploi familier de mots courants, ce qui constitue mon objet d'étude exclusif. Les emplois familiers sont à l'origine d'une polysémie particulière dont, parmi

7 Cf, par ex. : Jackie Schön, «Figement et régression ", in La locution : entre lexique, syntaxe et pragmatique, P. Fiala, P. Lafon, M.-F. Piguet (éd.), Paris, Public. de l'Inalf, coll. Saint-Cloud', Klincksieck, 1997, p. 333-345.

8 Je pense à l'appareil rhétorique, bien entendu...

9 Pour les aspects illocutoire et perlocutoire de la parole, on se référera aux ouvrages essentiels d'orientation pragmatique linguistique : Quand dire c'est faire de John Austin (trad. 1970), Les Actes de Langage de John Searle (trad. 1972) et Catherine Kerbrat-Orecchioni, notamment L'énonciation, de la subjectivité dans le langage, 1980.

10 "Emmerdant est un mot familier " donné en exemple dans le petit Robert 2000, tandis que dans celui de 1973, nous avons en exemple boulette pour bévue... Curieux, n’est-ce pas ?. 
les exemples les plus banals, l'andouille française. Sommes-nous à même de justifier le passage du sens premier du terme à son acception familière et quels éclaircissements nous seront ils fournis par les dictionnaires?

Pour une unité linguistique donnée, plusieurs acceptions ${ }^{11}$ peuvent être proposées, cas à distinguer de celui de plusieurs entrées pour des homonymes. Après des indications phonétiques, morphologiques, étymologiques ou de provenance de l'unité avec, en plus, des datations, les sens différenciés apparaissent, numérotés ${ }^{12}$. Tout cela est communément connu, ne nous y attardons pas davantage.

Outre ces indications, le dictionnaire fournit ce qu'il appelle des marques d'usage et ce sont celles qui nous intéressent ${ }^{13}$. Les dictionnaires n'offrent pas un modèle d'uniformité en la matière car, d'une édition à l'autre ou d'une maison d'édition à une autre, les marques peuvent varier ; elles oscillent, particulièrement, entre figuré (fg.), familier (fam.), populaire ( $p \circ p$.), vulgaire (vulg.), argotique (arg.), ou encore vieilli, régional (région.) ou dialectal (dial.) selon les cas. Rien de plus prévisible que le relevé des variantes entre une édition et une autre plus récente, en exemple : dans le Robert de 1972, ouvert au hasard, pour le substantif grue, on trouve en sens $2:$ : (1415) Fig. et pop. (des stations prolongées de la fille qui fait le pied de grue). Femme de mœurs légères et vénales. V. Prostituée. " tandis que l'édition de 2000 nous donne l'emploi comme vieilli, équivalent aussi à Prostituée et, en sus, valant insulte en grand rapport de sens ${ }^{14}$ avec Putain. Certes, il ne s'agit là que d'infimes nuances mais, ayant travaillé, antérieurement, la question et afin d'éviter de passer trop de temps à une recherche annexe, je choisis de renvoyer aux exemples plus probants que $\mathrm{j}$ 'avais antérieurement retenus ${ }^{15}$.

Ce qui ressort de ce survol, c'est le flottement qui entoure certaines de ces marques sinon toutes quant au contenu des vocables qui acquièrent un sens nouveau en emploi familier. Quelques exemples suffiront pour en observer des caractéristiques communes, soit $^{16}$ :

- andouille : 2 (1865). Fam. : Niais, imbécile. Quelle andouille, ce type!

- courge : 3 Fam. : Imbécile -----> gourde. Quelle courge!

11 Étant entendu que qui dit acception dit sens en emploi.

12 Cf., si besoin était, la Préface du Nouveau Petit Robert, édition remaniée et amplifiée sous la direction de Josette Rey-Debove et Alain Rey, éd. de juin 2000.

13 Cf. en particulier : F. Baider, E. Lamprou, M. Monville-Burston (éd.), La marque en lexicographie, Etats présents, voies d'avenir, Actes du Colloque international de L'Université de Chypre, Nicosie, oct. 2006, Lambert-Lucas, Limoges, 2011, col. « La lexicothèque ».

14 Je transcris littéralement l'expression du dictionnaire.

15 Jackie Schön, "Pour un traitement systématique des acceptions familières dans les dictionnaires ", in Hommage à Jacques Allières, M. Aurnague et M. Roché (éds), Anglet (France), Éd. Atlantica, 2002, vol. 2, p. 605-615.

16 Les exemples sont tous extraits du Petit Robert 2000 sauf noix tiré du Robert 1972. 
- cruche : 2 (1633). Fam. : Personne niaise, bête et ignorante -----> gourde, imbécile. Quelle cruche, ce type!

- gourde : 2 Fig et Fam. : Personne niaise, maladroite ------> cruche. Ce type est une gourde. ${ }^{17}$

- tarte: 3 (1895) Fam. (personne). Laid; sot et ridicule, peu dégourdi ------> cloche, cruche, tocard.

- cloche : 6 Fam. vieilli : Tête.

- poire : 4 (1896) Fam. : Personne qui se laisse tromper facilement, se laisse faire -.---> naïf. Quelle poire, ce type ! --.--> imbécile, sot.

Cette liste, limitée provisoirement aux seules unités nominales par souci de clarté d'exposition, n'est qu'indicative, d'autres exemples suivront au fur et à mesure des opportunités, l'essentiel étant de dégager la tonalité générale de ces équivalences, tonalité monotone, péjorative et imprécise.

\section{L'imprécision}

Chacune de ces épithètes mérite approfondissement. En effet, au flottement des marques entre elles semble correspondre l'interchangeabilité des définitions elles-mêmes avec le flou qui en résulte immanquablement. Où passe donc la différence entre s'entendre traiter de courge, d'andouille ou de tarte? Lequel de ces déterminants aurait le plus de chance de peser lourd devant un tribunal au cas où nous oserions y déposer une plainte pour insulte?

En outre, la péjoration qui accompagne chacun des exemples cités n'estelle pas déjà présente dans l'indexation qui signale une restriction de leurs usages allant de pair avec une stigmatisation sociale?

On pourra objecter que certaines expressions se situent à l'opposé des termes péjoratifs comme c'est le cas pour les hypocoristiques type : mon lapin, mon canard, ma puce... Mon chou reste un exemple presque trop beau tant il cumule à lui seul de traits caractéristiques de l'exemplification. N'illustret-il pas, à la fois, l'imprécision sémantique, l'importance du contexte, de la construction syntaxique, la tendance au figement sans oublier l'exploitation faite de l'image ${ }^{18}$ ?

Avant que de traiter, successivement, chacun de ces points, notons que les usages tant hypocoristiques que méprisants, voire injurieux, d'une expression ressortissent exactement au même mécanisme dont la description suivra. Insulte ou mot d'amour, même procédé, même combat !

17 Le lexicographe ajoute en remarque : «Plus courant en parlant d'une femme ».

18 Le propre des exemples et la difficulté d'analyse en leurs composants ne résident-ils pas en l'extrême condensation de ces derniers ? Ainsi trouve-t-on à côté de mon chou : bête comme chou, être dans les choux, entrer dans le chou, faire chou blanc, faire ses choux gras, une feuille de chou (en tant que journal de qualité médiocre), etc. Mais, s'il fallait attribuer un signifié précis à ces choux-là sur lequel nous arrêterions-nous ? 
L'imprécision, donc, caractérise de tels emplois, le fait est si patent qu'il paraît superflu d'y insister. Cependant, là encore, des objections peuvent s'élever car il est vrai qu'il existe des cas de fixation d'un sens plutôt qu'un autre pour certains vocables, obéissant en cela au principe fonctionnel de différenciation cher à Henri Frei ${ }^{19}$. Ainsi la naïveté s'accroche-t-elle à la poire (pourquoi ?) tandis que la nouille évoquera facilement la mollesse, ceci est indéniable et presque inévitable mais qu'en est-il de la tarte et de la laideur mentionnée dans le dictionnaire, la laideur, la sottise ou le ridicule accolés au terme comme figurant ci-dessus ? Si une enquête était menée ${ }^{20}$, pour préciser ce qui est supposé être dit et/ou entendu lors d'un traitement de tarte! à l'endroit d'une personne, est-il si sûr que la laideur figurerait parmi les réponses?

Avec la sensation de résoudre une énigme de roman policier, j'en avance d'abord la solution avant que d'en démonter les rouages. Il s'avère que tant la tarte que la gourde, la bécasse ou le ballot... sont des formes vides de sens spécifique, elles n'en acquièrent que de par leur emploi particulier. Cet emploi est, effectivement, doté d'une signification, celle de l'affect qu'exprime celui qui use de cet emploi. Les petits " noms d'oiseaux " qu'actualisent les locuteurs dans des discours adressés à autrui ne renvoient à aucun concept mais leur emploi traduit leurs affects. Or, il convient d'ajouter qu'autant l'imagination se montre fertile pour multiplier les termes d'adresse affectifs, autant la palette des moyens d'exprimer la subjectivité est réduite ; elle se résume à être positive ou négative, à dire " j'aime ou je n'aime pas ", je suis pour ou je suis contre. Alors que nous disposons d'une richesse lexicale inépuisable pour décrire nos sentiments, nos impressions, nos états d'âme ou d'esprit, nous voilà censurés en permanence par notre communauté linguistique sans que nous y prenions garde et bien que nous ayons été dûment avertis de la surveillance par la marque fam.

\section{La polysémie familière}

\section{La métaphore}

D'aucuns ${ }^{21}$ allèguent un processus métaphorique pour éclairer la créativité familière. La pomme, la cruche, la courge, la noix... se formeraient par quelque analogie entre la forme ronde et la face humaine. Un navet qualifiant un mauvais spectacle se justifierait par la fadeur du légume tandis qu'un pastis se baserait sur l'idée du mélange pour convoquer celle d'une complication, admettons! Que dire d'un mufle, d'un cornichon, d'un ballot, d'une

19 Ce principe devrait répondre au besoin fondamental de clarté chez l'homo loquens, Henri Frei, La grammaire des fautes, 1929, ré-éd. 2007, Rennes, Ennoïa.

20 Ce que je n'ai pas fait...

21 Dont C. Kerbrat-Orecchioni autant qu'il m'en souvienne. 